\title{
Third International Symposium on Innovation and Information and Communication Technology (ISIICT 2009)
}

\section{5 - 17 December 2009}

Philadelphia University, Amman, Jordan 


\section{Abstract}

The scope of the third International Symposium on Innovation in Information and Communication Technology is fairly wide, since it is intended to serve as a forum for discussing the wide range of issues that are raised by today's information and communication technology, as well as their evolution and their paradigm shifts.

The programme for this symposium is divided into seven areas of interest: data mining and web information retrieval, software engineering, performance evaluation, computer security, information systems and ERP, e-applications, and diagnosis and image processing applications.

This conference is sponsored by:

\section{$\underline{\text { IEEE }}$}

The Higher Council for Science and Technology

BCS, The Chartered Institute for IT 


\section{Full Synopsis}

The scope of the third International Symposium on Innovation in Information and Communication Technology is fairly wide, since it is intended to serve as a forum for discussing the wide range of issues that are raised by today's information and communication technology, as well as their evolution and their paradigm shifts.

The programme for this symposium is divided into seven areas of interest: data mining and web information retrieval, software engineering, performance evaluation, computer security, information systems and ERP, e-applications, and diagnosis and image processing applications.

The track of data mining and web information retrieval includes three papers that are divided in the following topics of interest: learning from web, textual data, and web usage mining.

The track of software engineering includes four papers that are divided in the following topics of interest: dynamic and structural pattern, software evolution, real-time embedded systems, and web service applications. The track of performance evaluation includes two papers that are divided in the following topics of interest: performance of routers and performance of generic deployment platform.

The track of computer security includes two papers that are divided in the following topics of interest: cryptographic protocols and wired and wireless networks. The track of information systems and ERP includes two papers that are divided in the following topics of interest: validation of the IS-impact model and critical success factors of ERP.

The track of e-applications includes three papers that are divided in the following topics of interest: ebusiness value, G2C acceptance, and wireless networks. The track of diagnosis and image processing applications includes three papers that are divided in the following topics of interest: distributed systems diagnosis, turbine aided diagnostic, and image segmentation.

In addition to these peer-reviewed papers two invited keynote speakers were presented at this symposium: Professor Fairouz Kamareddine spoke about 'The Computerisation of Information: The Challenges and the Pitfalls', and Professor Odej Kao spoke about 'Resource Management and Trust in On-Demand Computing'.

We are pleased by the international diversity of the symposium; authors hail from no less than seven countries, including Algeria, Australia, France, Jordan, Malaysia, Tunisia and The United Kingdom.

We are very grateful to the programme committee members and reviewers for their help in assessing and selecting papers among those that are submitted. Also we gratefully acknowledge the support of the organising committee who, in addition to their organising responsibilities, has offered us a great deal of technical support to manage the flow of papers and reviews. The technical support of IEEE, the technical and financial support of BCS, The Chartered Institute for IT, and the financial support of The Higher Council for Science and Technology were very appreciated and helped the success of this symposium. 


\section{Committee}

General Chair

Khaldoun Batiha. Philadelphia University Chair, Jordan

\section{Programme Committee Chair}

Fairouz Kamareddine, Heriot-Watt University, Scotland

\section{Organising Committee Chair}

Said Ghoul. Philadelphia University Chair, Jordan

\section{Programme Committee}

\section{Chair}

Fairouz Kamareddine, Heriot-Watt University, Scotland.

\section{Members}

Abdelmajid Benhamadou, Tunisia,

Adam Belloum, University of Amsterdam, Netherlands

Aladdin Ayesh, De Montfort University, UK

Ana Cavalli, Institut Telecom/ Telecom \& Management, France

Bruno Monsuez, ENSTA/UEI Paris, France

Christine Choppy, Paris 13 University, France,

Cyrille Bertelle, LITIS-University of Du Havre, France

Dmitry A. Zaitsev, Odessa National Academy of Telecommunications, Ukrain

Fernando Orejas, Technical University of Catalonia, Spain,

Fouad Badran, CNAM, France 
Gerard Duchamp, Paris 13 University, France

Hassan B. Diab, American University of Beirut, Lebanon

Hisham Al-Mubaid, University of Houston Clear Lake, USA

Joan. LU, Huddersfield University, UK

Kamel Barkaoui, CNAM, France

Karim Djemame, University of Leeds, UK

Kevin Daimi, University of Detroit Mercy, USA

Mourad Debbabi, Concordia University, Montreal, Canada

Nashat Mansour, Lebanese American University, Lebanon

Noureddine Belkhatir, Grenoble University, France,

Odej Kao, Technical University Berlin, Germany

Patrice Moreaux, LISTC - Polytech'Savoie, France

Rachida Dssouli, Concordia University, Canada.

Said Bettayeb , University of Houston - Clear Lake, USA

Sasikumaran Sreedharan, King Khalid University, Saudi Arabia

This conference was edited by:

General Chair

Tom McEwan Edinburgh Napier University, UK

Technical Programme Chairs

David Benyon Edinburgh Napier University, UK

Sriram Subramanian University of Bristol, UK

Organisation Chair

Lucia Terrenghi Vodafone

Programme Committee Members

Anupam Agrawal IIIT Allahabad, India

Stephen Brewster University of Glasgow, UK

Alan Dix Lancaster University, UK

Sumantra Dutta Roy IIT Delhi, India 
Anirudha Joshi IIT Bombay, India

John Knight Vodafone

Ann Light Queen Mary University of London, UK

Sriganesh Madhvanath HP Labs, India

Tom McEwan Napier University, UK

Hema Murthy IIT Madras, India

Andy Smith Thames Valley University, UK

Lucia Terrenghi Vodafone

Rama Vennelakanti HP Labs, India 


\section{Papers:}

\section{Session 1: Data Mining \& Web Information Retrieval}

Nesrine Ben Mustapha, and Hajer Baazaoui Zghal, Marie-Aude Aufaure, and Henda Ben Ghezala Survey on Ontology learning from Web and open issues http://dx.doi.org/10.14236/ewic/ISIICT2009.1

Nadia Zerida and Khaldoun Zreik Combining Evidence with Logic and Preferences to Learn Relations from Structured Few Sparse Textual Data http://dx.doi.org/10.14236/ewic/ISIICT2009.2

Alaa H. AL-Hamami, Mohammad A. AL-Hamami, and Soukaena H. Hashem Applying Packets Meta data for Web Usage Mining http://dx.doi.org/10.14236/ewic/ISIICT2009.3

\section{Session 2: Software Engineering}

Nadia Bouassida and Hanene Ben-Abdallah Design improvement through dynamic and Structural pattern identification http://dx.doi.org/10.14236/ewic/ISIICT2009.4

Labiba Souici-Meslati, Djamel Meslati and Seifeddine Mili Software Evolution and Natural Processes: A Taxonomy of Approaches http://dx.doi.org/10.14236/ewic/ISIICT2009.5

Mustapha Bourahla Modeling and Verification of Real-Time Embedded Systems http://dx.doi.org/10.14236/ewic/ISIICT2009.6

Dhikra Kchaou, Nadia Bouassida, and Hanene Ben-Abdallah WS-UML: A UML Profile for Web Service Applications $\quad$ http://dx.doi.org/10.14236/ewic/ISIICT2009.7

\section{Session 3: Performance Evaluation}

Hussein Al-Bahadili, Jafar Ababneh and Fadi Thabtah Derivation of an Analytical Model for Evaluating the Performance of a Multi-Queue Nodes Network Router

http://dx.doi.org/10.14236/ewic/ISIICT2009.8 


\section{Session 4: Computer Security}

Hasan Al-Refai Evaluation Technique in the Spicalculus for Cryptographic Protocols http://dx.doi.org/10.14236/ewic/ISIICT2009.9

Anthony C. Ijeh, Allan J. Brimicombe, David S. Preston, and Chris Imafidon Security Measures in

Wired and Wireless Networks http://dx.doi.org/10.14236/ewic/ISIICT2009.10

\section{Session 5: Information Systems \& ERP}

Ahmad A. Rabaa'l Validating The IS-Impact Model At Queensland University Of Technology (QUT):

Part A http://dx.doi.org/10.14236/ewic/ISIICT2009.11

Ahmad A. Rabaa'l Identifying Critical Success Factors of ERP Systems at the Higher Education

Sector http://dx.doi.org/10.14236/ewic/ISIICT2009.12

\section{Session 6: E-applications}

Ramlah Hussein, Norshidah Mohamed, Abdul Rahman Ahlan, Murni Mahmud and Umar Aditiawarman G2C Acceptance in Malaysia: Trust, Perceived Risk and Political Efficacy http://dx.doi.org/10.14236/ewic/ISIICT2009.13

Rania A. Bakeir, Emad Abu-Shanab and Shadi Al Biss Applying E-marketing Methodologies In The Mobile Industry: The Case of Orange - Jordan http://dx.doi.org/10.14236/ewic/ISIICT2009.14

\section{Session 7: Diagnosis and Image Processing Applications}

Hammadi Bennoui, Allaoua Chaoui and Kamel Barkaoui Exploiting P-invariant analysis for distributed systems diagnosis based on interacting behavioral Petri nets

http://dx.doi.org/10.14236/ewic/ISIICT2009.15

Klai Sihem and Khadir Med Tarek Data based Ontology Construction Coupled to Expert System for Steam Turbine Aided Diagnostichttp://dx.doi.org/10.14236/ewic/ISIICT2009.16 
Safia Djemame, Mabrouk Nekkache and Mohammed Batouche A Multi-Agent System for Image Segmentation A Bio-Inspired Approach http://dx.doi.org/10.14236/ewic/ISIICT2009.17 